\title{
Influenza A virus antagonizes type I and type II interferon responses via SOCS1-dependent ubiquitination and degradation of JAK1
}

Yinping Du

Yangzhou University

Fan Yang

Yangzhou University

Qiuxia Wang

Yangzhou University

Nuo Xu

Yangzhou University

Yizhang Xie

Yangzhou University

Sujuan Chen

Yangzhou University

Tao Qin

Yangzhou University

Da-Xin Peng ( $\nabla$ pengdx@yzu.edu.cn )

Yangzhou University

\section{Research}

Keywords: IAV, JAK1, IFN, SOCS1, degradation

Posted Date: January 28th, 2020

DOI: https://doi.org/10.21203/rs.2.22023/v1

License: (c) (1) This work is licensed under a Creative Commons Attribution 4.0 International License.

Read Full License

Version of Record: A version of this preprint was published at Virology Journal on June 12th, 2020. See the published version at https://doi.org/10.1186/s12985-020-01348-4. 


\section{Abstract}

BACKGROUND Although influenza A virus (IAV) employs diverse strategies to evade IFN responses by inhibiting the synthesis of IFN, how IAV regulates signaling downstream of IFN is incompletely understood.

METHODS In this study, we used Western blot-based protein analysis coupled with RT-qPCR, overexpression and RNA interference to investigate the regulation of JAK1 by IAV infection in 293T cells.

RESULTS The results indicated that JAK1 was ubiquitinated and degraded, resulting in inhibition of type I and type II IFN responses, demonstrating that IAV antagonizes the IFN-activated JAK/STAT signaling pathway by inducing the degradation of JAK1. Furthermore. IAV infection upregulated the suppressor of cytokine signaling (SOCS) protein SOCS1, and SOCS1 mediated the ubiquitination and degradation of JAK1.

CONCLUSION Collectively, our findings suggest that IAV infection induced SOCS1 expression promotes JAK1 degradation, which in turn inhibits host innate immune responses.

\section{Introduction}

The emergence of influenza A virus causing significant morbidity and mortality in people remains a global health concern. Influenza A virus naturally circulate in the wild bird population, such as waterfowl and ducks, and can spill over to other species, including humans[1]. Outbreaks of avian influenza virus such as H5N1, H7N9, and H9N2 virus have caused high morbidity and mortality rates in humans, raising the risk for the occurrence of influenza pandemics[2-4]. Antiviral drugs are available for treating influenza, but numerous strains of IAV are resistant, presumably due to mutation. Thus, identifying mechanisms for IAV regulation of host immunity and designing new therapeutic strategies are important to effectively control influenza.

Influenza virus infection can be sensed by host cellular pathogen recognition receptors (PRRs), which in turn activate downstream signaling cascades and then induce the expression of cytokines, including interferons (IFNs)[5]. IFNs are a superfamily of cytokines which are classified into type I, type II, and type III subtypes. IFNs and interferon-stimulated genes (ISGs) establish a crucial line of antiviral defense, inhibiting virus replication and restricting the spread of viruses[6]. After being secreted, the IFNs bind to the cognate IFN receptors to initiate the JAK/STAT signaling pathway, involving tyrosine kinases of JAK family and transcription factors of STAT family[6, 7]. Activation of JAK/STAT pathway leads to the induction of various ISGs, and some ISGs have direct anti-influenza virus activities[8]. Previous studies using IFNs receptor or STAT1 gene knockout mice have demonstrated the importance of IFNs response to anti-influenza defense[9, 10].

It is not well understood how IAV regulate the IFN induced JAK/STAT signaling pathway. It was reported that IAV downregulate IFN receptors level upon infection, and then inhibit the antiviral activity of IFNs[11]. 
IAV infection induced SOCS1 can inhibit the activity of STAT1[12]. However, it is unknown whether and how IAV regulate the JAK1 protein downstream of IFNs receptor. Some viruses induce the degradation of JAK1, and then inhibited the IFNs stimulated antiviral and immunoregulatory activity. In this study, we investigated whether IAV infection regulates JAK1.

We found that IAV infection significantly downregulated the protein level of JAK1. IAV infection facilitated the ubiquitination of JAK1 to promote its degradation. Rescued JAK1 expression can restore the IFNs induced phosphorylation of STAT1 and the expression of ISGs. Those results indicated that IAV facilitate its replication by inducing the degradation of JAK1 during infection. We further show that IAV infection upregulated SOCS1 expression, and SOCS1 mediated JAK1 ubiquitination and proteasome dependent degradation. These data extend our knowledge of influenza pathogenesis and suggest new therapeutic targets for treating influenza.

\section{Materials And Methods}

\section{Virus and cells}

Three Influenza A virus isolates A/mallard/Huadong/S/2005 (H5N1)[13], A/chicken/Jiangsu/WJ14/2015 (H7N9)[14] and A/chicken/Taixing/10/2010 (H9N2)[15] were used in this study. Viruses were amplified in 10-day-old specific-pathogen-free (SPF) chicken embryonated eggs. Virus yields were quantified using TCID50 assays on MDCK cells. After adsorption at $37{ }^{\circ} \mathrm{C}$ for $1 \mathrm{~h}$ in $5 \% \mathrm{CO}_{2}$, the virusinfected MDCK cells were maintained in MEM containing $1 \%$ BSA and $0.5 \mu \mathrm{g} / \mathrm{ml}$ tosylphenylalanyl chloromethyl ketone (TPCK)-treated trypsin (Sigma Chemical Co., St. Louis, Mo.). Human lung epithelial A549 cells, human embryonic kidney 293T cells, and MDCK cells were cultured in Dulbecco's modified Eagle's medium (DMEM; Gibco) with $10 \%$ FBS (Gibco) and penicillin (100 U/ml) - streptomycin (100 $\mathrm{ug} / \mathrm{ml}$ ) (Invitrogen).

\section{Reagents and antibodies}

Cycloheximide (CHX; Sigma-Aldrich), anti-DYKDDDDK (FLAG) G1 Affinity Resin (GenScript), phenylmethylsulfonyl fluoride (PMSF) (Gold Bio), immunoprecipitation (IP) lysis buffer (Thermo Scientific), proteasome inhibitor $\mathrm{MG} 132$ (Selleck chem), $\mathrm{NH}_{4} \mathrm{Cl}$ (Selleck chem), and recombinant human IFN-a2 and IFN- $\gamma$ (GenScript) were purchased from the indicated manufacturers. Antibodies against JAK1, STAT1, phospho-STAT1, and $\beta$-actin were purchased from Sigma; antibodies against SOCS1 and SOCS3 were purchased from GeneTex, antibodies against influenza virus NP, M1, and NS1 were purchased from GeneTex; antibodies against DYKDDDDK (FLAG) tag and HA tag were purchased from Cell Signaling Technology. Human SOCS1 siRNAs si-1, GCAUCCGCGUGCACUUUCAdTdT, and si-2, CUACCUGAGCUCCUUCCCCdTdT were synthesized by Gene Pharma.

\section{Constructs and transfection}


The human JAK1 gene was amplified using reverse transcription PCR (RT-PCR), and pcDNA3.1(+)-His and p3xFlag CMV-14 were used to construct plasmids encoding JAK1. Ub gene was amplified using reverse transcription PCR (RT-PCR) and cloned into the vector pCDNA3.1(+)-HA to construct HA-Ub. For transient expression in 293T cells, cells were transfected with plasmids using PolyJet In Vitro DNA Transfection Reagent (Signa gene), according to the protocol recommended by the manufacturer. Briefly, the transfection complex was prepared at a ratio of PolyJet to DNA of 3:1. The 293T cells were incubated with the transfection complex at $37^{\circ} \mathrm{C}$ for indicated times. For siRNA transfection, 293T cells plated onto a 6-well plate were transfected with siRNAs targeting SOCS1 at a concentration of $100 \mathrm{pmol} / \mathrm{ml}$ using Lipofectamine 2000 (Thermo Fisher), according to the protocol recommended by the manufacturer.

\section{Western blot analysis}

Cells were lysed in the $2 x$ sample buffer (Beyotime) and heated at $100{ }^{\circ} \mathrm{C}$ for $10 \mathrm{~min}$. Equal amounts of protein samples were resolved on a $10 \%$ sodium dodecyl sulfate-polyacrylamide gel electrophoresis (SDS-PAGE) gel and transferred to nitrocellulose membrane (Bio-Rad). Membrane-bound proteins were incubated with specific antibodies and detected using an enhanced chemiluminescence substrate (Thermo Scientific). All the data presented were repeated at least twice in independent experiments.

\section{Real-time PCR}

Total cellular RNA was purified using Trizol Reagent (Sigma-Aldrich) according to the manufacturer's instructions and was treated with DNase I (Thermo Scientific) to remove contaminated DNAs. The RNA was reverse transcribed using oligo-18dT (Invitrogen), and the resulting CDNA was then analyzed by realtime quantitative PCR (qPCR) using gene-specific primers. Primers for human JAK (5'-CTT TGC CCT GTA TGA CGA GAA C-3' and 5'-ACC TCA TCC GGT AGT GGA GC-3'), Mx1 (5'-GTT TCC GAA GTG GAC ATC GCA3' and 5'-CTG CAC AGG TTG TTC TCA GC-3'), ISG56 (5'-AGA AGC AGG CAA TCA CAG AAA A-3' and 5'-CTG AAA CCG ACC ATA GTG GAA AT-3'), TAP-1 (5'-TGT GAC AAG GTT CCC ACT GCT TAC3' and 5'-GGC TGT GGC CTA TGC AGT CA-3'), LMP-2 (5'-GCA TAT AAG CCA GGC ATG TCT CC-3' and 5'-AGC TGT AAT AGT GAC CAG GTA GAT GAC-3'), SOCS1 (5'-GAC ACT CAC TTC CGC ACC T-3'and 5'-GAA GAA GCA GTT CCG TTG G-3'), SOCS3 (5'-GCA GGA GAG CGG ATT CTA CT-3' and 5'-ACG CTC AAC GTG AAG AAG TG-3' )were used. QPCRs were performed with SYBR green I chemistry using a Step One Plus Real-Time PCR instrument. The comparative $\mathrm{Ct}[2(-\Delta \Delta \mathrm{Ct})]$ method was used to analyze gene expression, and genes quantities were normalized to the housekeeping gene (GAPDH, 5'-TCATCATCTCTGCCCCTTCT-3' and 5'GTCATGAGTCCCTCCACGAT-3').

\section{Immunoprecipitation and ubiquitination assay}

For detection of ubiquitination of JAK1 during IAV infection, 293T cells were transfected with FLAG-JAK1 $(1 \mu \mathrm{g})$ and tHA-Ub $(0.5 \mu \mathrm{g})$. At $24 \mathrm{~h}$ post transfection, cells were left uninfected or infected with IAV at a multiplicity of infection (MOI) of 1 for an additional $18 \mathrm{~h}$. For IP experiments, these cells were lysed with IP lysis buffer containing protease inhibitor (PMSF, $1 \mathrm{mM}$ ) and incubated with $20 \mu$ Anti-DYKDDDDK G1 affinity resin overnight under rotation at $4{ }^{\circ} \mathrm{C}$. The beads were washed three times with IP lysis buffer. The 
beads were washed, and the precipitates were analyzed by Western blotting. The experiments were independently repeated twice with similar results.

\section{Statistical analysis}

Prism version 5.0 (GraphPad) was used for data analysis. Statistical significance was analyzed using Student's $t$ test, and comparisons of experimental groups were estimated using one-way ANOVA analysis of variance. A p value less than 0.05 was considered to be significant.

\section{Results}

\section{Influenza A virus infection down regulates JAK1.}

To investigate the regulation of JAK1 during influenza A virus infection, JAK1 protein abundance was quantified using Western blotting. We infected cells with three different strain of influenza viruses: A/mallard/Huadong/S/2005 (H5N1), A/chicken/Jiangsu/WJ-14/2015 (H7N9) and A/chicken/Taixing/10/2010 (H9N2). Following all subtypes of IAV infection, the protein level of JAK1 was strongly decreased in both A549 cells, 293T cells and MDCK cells (Fig. 1A). IAV infection-induced JAK1 downregulation was time- and virus dose-dependent, and the protein level of JAK1 was inversely correlated with the replication of IAV, as assayed by the expression of viral NP in infected cells (Fig. 1B and 1C). These data demonstrate that IAV infection significantly downregulates the protein level of JAK1.

\section{IAV infection induces the ubiquitination and proteasome degradation of JAK1.}

We determined the stage of JAK1 downregulation during IAV infection. First, the mRNA level of JAK1 in IAV infected cells and control cells were detected using real-time quantitative PCR. IAV infection had no effect on the mRNA level of JAK1 (Fig. 2a), suggesting that the inhibition of JAK1 expression occurred at the post-transcriptional stage. Then we treated IAV infected cells and control cells with the translation inhibitor cycloheximide (CHX) and then detected the protein stability of JAK1. JAK1 degraded faster in IAV infected cells than in control cells, suggesting that IAV infection accelerates JAK1 protein degradation (Fig. 2b). As the ubiquitin proteasome system was reported to mediate the degradation of JAK1[16], we determined if IAV infection induces JAK1 ubiquitination. To detect the ubiquitination of JAK1, Flagtagged JAK1 and HA-tagged Ub were transiently overexpressed in 293T cells, and the ubiquitination of JAK1 was assessed using immunoprecipitation and Western blotting. IAV infection increased the ubiquitination level of JAK1, indicating that JAK1 ubiquitination is induced by IAV infection (Fig. 2c). Proteasome-dependent and lysosome-dependent protein degradation pathway are two distinct protein degradation mechanisms. We sought to investigate the JAK1 degradation pathway induced by IAV infection. Following infection with IAV, cells were treated with MG-132 (proteasome inhibitor) or $\mathrm{NH}_{4} \mathrm{Cl}$ (lysosome inhibitor). The degradation of JAK1 induced by IAV was repressed by MG-132 but not by $\mathrm{NH}_{4} \mathrm{Cl}$, suggesting that proteasome-dependent pathway mediated the degradation of JAK1 during IAV infection (Fig. 2d). 


\section{IAV infection induces cell less responsive to type I and type II IFNs.}

Jak1 and the downstream transcription factors are required for the cellular responses to IFNs. Given the ability of IAV to reduce the protein level of JAK1, we sought to gain insight into the influence of IAV in IFNs mediated signaling pathway. We first investigated the activation of transcription factor STAT1 in response to type I and type II IFNs in the presence or absence of IAV infection. Treatment with recombinant human IFN-a or IFN-y induced STAT1 activation, eliciting high levels of pSTAT1 (Fig. 3a and 3b). However, IAV infection strongly inhibited STAT1 phosphorylation after stimulation with IFN-a or IFN- - , and this inhibition was correlated with the downregulation of JAK1 (Fig. 3a and 3b). IFNs treatment can induce the expression of antiviral ISGs through JAK/STAT pathway, and ISGs play key rule in and immune regulation and controlling virus replication[6,17]. We then determined whether IAV infection induced JAK1 degradation regulates the expression of ISGs. The transcription profiling of ISGs in response to IFN- $a$ and IFN- $\gamma$ was analyzed using qPCR. IAV infection substantially inhibited IFN- $a$ induced expression of ISG56 and Mx1, as well as IFN-y triggered Tap-1 and Lmp-2 (Fig. 3c and 3d). Collectively, these results indicate that IAV infection induces JAK1 degradation and decreases cellular sensitivity to IFNs.

\section{Rescuing JAK1 expression inhibits IAV replication.}

To further demonstrate the association of JAK1 degradation and IFNs response attenuation during IAV infection, 293T cells were transfected with JAK1-His or control vector. Most of the overexpressed JAK1 was degraded during IAV infection, and JAK1 overexpression inhibited the replication of IAV, indicating that JAK1 degradation induced by IAV infection was beneficial to virus replication (Fig. 4a). JAK1 overexpression partially restored the activation of STAT1 in response to IFN- $a$ and IFN- $\gamma$ in the presence of IAV infection (Fig. 4b and 4c). The mRNA level of ISGs were also increased by JAK1 overexpression (Fig. $4 \mathrm{~d}$ and $4 \mathrm{e}$ ). IAV infection can also inhibit the IFNs response by other ways, such as by decreasing the protein level of IFN receptors[11]. Transient expression of JAK1 cannot fully restore the phosphorylation of STAT1 as well as the expression of ISGs. Collectively, overexpression of JAK1 can partly restore cellular response to type I and type II IFNs and display anti influenza activity, suggesting that JAK1 degradation play a critical role in attenuating the antiviral activity of IFNs during IAV infection.

\section{IAV infection induced SOCS1 mediate the degradation of JAK1}

SOCSs family proteins play an important role in negatively regulation JAK/STAT signaling pathway[18, 19]. Previous studies have revealed that IAV infection upregulates SOCS1 and SOCS3, and SOCS $1 / 3$ antagonist peptide can protect mice against lethal influenza infection[20]. IAV infection induced SOCS1 can inhibit the phosphorylation of STAT1[12]. SOCS1 and SOCS3 also target JAK1 for degradation[21, 22]. Therefore, we investigated whether SOCS1 and SOCS3 are involved in the degradation of JAK1 during IAV infection. The protein level of SOCS1 was significantly increased during IAV infection, and the protein level of SOCS3 was not changed (Fig. 5a). IAV infection also upregulated the mRNA level of SOCS1 (Fig. 5b). To further verify the correlation of SOCS1 protein expression and JAK1 degradation, SOCS1 protein level in 293T cells were knocked down using siRNA (Fig. 5c). In SOCS1 knockdown cells, 
the protein level of JAK1 was notably higher than that of control cells (Fig. 5c), indicating that SOCS1 directly mediated JAK1 degradation during IAV infection. Importantly, knocked down of SOCS1 inhibited the expression of virus protein NP upon IAV infection (Fig. 5c). These results indicate that IAV infection induced SOCS1 is a negative regulator of cellular antiviral activity, and SOCS1 plays an important role in downregulating the protein level of JAK1. To determine if SOCS1 mediates JAK1 ubiquitination and degradation, 293T cells were transfected with siRNA targeting SOCS1, then transfected with Flag-tagged JAK1 and HA-tagged ubiquitin, and then infected with IAV. SOCS1 knockdown attenuated the ubiquitination of JAK1 during IAV infection (Fig. 5d). Collectively, these results indicate that SOCS1 mediates the ubiquitination and degradation of JAK1 during IAV infection.

\section{Knock down of SOCS1 rescues IFNs response}

To further ascertain the importance of SOCS1 in inducing JAK1 degradation during IAV infection, SOCS1 were knocked down using siRNA before testing the cellular response to IFNs SOCS1 knockdown rescued the protein level of JAK1 as well as the phosphorylation of STAT1 induced by type I and type II IFNs (Fig. $6 a$ and $6 \mathrm{~b}$ ). To further investigate the association between SOCS1 and IFNs response inhibition, the expression level of ISGs were detected by real-time PCR; SOCS1 knockdown enhanced the expression of ISGs induced by IFNs (Fig. 6c and 6d), indicating that SOCS1 knock down can enhance the IFNs induced ISGs expression during IAV infection. Collectively, these results illustrate that SOCS1 expression induced by IAV infection can downregulate the protein level of JAK1, making cells less responsive to IFNs.

\section{Discussion}

Zoonotic strains of influenza A virus remain important threats to global health, especially those strains that cause significant morbidity and mortality. Upon virus infection, interferon responses are induced in host cells to prevent viral replication, but IAV can evade cellular IFN response to propagate in host cells. Influenza A virus has evolved diverse strategies to inhibit the synthesis of IFN, but how IAV evades the down-stream signaling pathway of IFNs is not well elucidated. In this study, we demonstrated that influenza virus infection induced the degradation of JAK1, and then inhibited the cellular response to type I and type II IFNs. Influenza virus infection induced SOCS1 mediated the ubiquitination and degradation of JAK1, attenuating the cellular responsiveness to type I and type II IFNs.

JAK1 is a key regulator of interferon response and immune cell activation. Upon receptor ligation by IFNs, interferon receptors activate the catalytic activities of receptor associated Janus Kinase (JAK) family of tyrosine kinases to transduce signals[23]. Tyrosines in the cytoplasmic regions of the receptors are phosphorylated by activated JAKs, subsequently recruiting and phosphorylating STAT factors. Activated STATs translocate into the nucleus and then function as transcription factors, regulating the transcription program of ISGs. ISGs play an important role in cleaning the infection by directly inhibiting virus replication and regulating the antiviral immune functions. We found that IAV induced degradation of JAK1 and attenuated cells responsive to IFNs. Thus, JAK1 elimination induced by IAV could allow the virus to evade IFN-mediated antiviral activities. JAK1 can also mediate intracellular signaling from 
multiple cytokine receptors, such as IL-6 family cytokines, IL-10 family cytokines, IL-2, IL-4, IL-7, IL-9, IL-15, IL-21, and IL-27[24]. Some of those cytokines are involved in the "cytokine storm" induced by IAV infection and play diverse roles in influenza immune responses. Further studies should be done to evaluate the importance of JAK1 degradation induced by IAV infection.

Many viruses attenuate cellular responses to IFNs by targeting proteins of IFNs signaling pathway for degradation. On the receptor level, interferon receptors degradation are induced during IAV, HSV, HCV, and VSV[25]. IFN activated STAT1 is a target for ubiquitylation and degradation during multiple viruses infection[26]. Human Metapneumovirus (hMPV) enhances the proteasomal degradation of JAK1 protein in A549 cells, and then inhibits IFN- $\beta$ stimulated antiviral and immunoregulatory activity[27]. Foot-andmouth disease virus (FMDV) to degrades JAK1 via a lysosomal pathway to inhibit IFN-y signaling transduction pathway[28]. Zika virus (ZIKV) suppresses JAK/STAT signaling by targeting JAK1 for proteasomal degradation, impairing interferon mediated antiviral response[29]. Human cytomegalovirus (HCMV) induces proteasome-dependent degradation of Jak1, inhibiting IFN-a signal transduction pathway[30]. Although influenza A virus was previously reported to reduce the protein level of JAK1[31], how the virus might inhibit JAK1 and the influence of JAK1 downregulation during IAV infection were not assessed. We found that IAV infection induces JAK1 degradation in several cell types.

SOCS box proteins are the most well-described negative regulation factors of cytokine-induced signaling. There are eight numbers in the SOCS family, and SOCS1 is the most extensively studied inhibitor of JAK/STAT pathway.. SOCS1 can directly bind to IFNAR1 and IFNGR1 and then inhibit IFNs mediated activities[32,33]. SOCS1 can also directly interact with JAKs, resulting in inhibition of JAKs activity[34]. SOCS1 targets JAK1 for proteasomal degradation $[35,36]$. Several recent studies showed that IAV induces expression of SOCS1 to inhibit STAT activation, which suppressed the IFNs activation[12, 20]. Consistent with these observations, our data show that IAV infection induced expression of SOCS1, and SOCS1 mediated the degradation of JAK1. During IAV infection, overexpression of JAK1 or knockdown of SOCS1 can partially restore the activation of STAT1 induced by IFNs, enhancing the transcription of antiviral ISGs.

Protein ubiquitylation is crucial for regulating IFNs response via inducing protein degradation. Protein ubiquitylation involves conjugation of ubiquitin to the substrate proteins. SOCS box proteins can function as adaptors of the $\mathrm{E} 3$ ubiquitin ligase complex through interacting with Elongin B/C complex, and then target the substrate proteins for ubiquitination and degradation[37]. Several studies showed that SOCS1 targeted JAK1 to the proteasome for degradation[35, 36]. Recently, the interaction of SOCS1, JAK1, and Elongin $B / C$ was elucidated, demonstrating regulation of JAK1 by SOCS1[34]. We found that IAV induced SOCS1 mediates the ubiquitination and degradation of JAK1, resulting in inhibition of IFNs activity. A previous study showed that E3 ubiquitin ligase RNF125 bound to JAK1 and promoted its ubiquitination and degradation[16]. Nedd4 family E3 ubiquitin ligases can mediate the degradation of JAK1 and restrict cytokine signaling to limit $T$ cells expansion[38]. It will be interesting to further investigate whether RNF125, Nedd4 family E3 ubiquitin ligases, and other E3 ubiquitin ligases, participate in SOCS1 mediated degradation of JAK1 during IAV infection. 


\section{Conclusion}

Taken together, our results revealed that IAV infection induced SOCS1 attenuate cellular responses to IFNs by targeting JAK1 for degradation. Investigating the JAK1 elimination induced by IAV could help us better understand the pathogenesis of IAV and may provide new therapeutic targets for treating influenza.

\section{Abbreviations}

IAV Influenza A virus

IFN Interferon

JAK1 Janus kinase 1

STAT1 Signal transducer and activator of transcription 1

SOCS1 suppressor of cytokine signaling 1

ISG Interferon stimulated gene

\section{Declarations}

\section{Ethics approval and consent to participate}

Not applicable

Consent for publication

Not applicable

\section{Availability of data and materials}

All data generated or analysed during this study are included in this published article

\section{Competing interests}

The authors declare that they have no competing interests

\section{Funding}

This work was supported by the following funding sources: The National Key R\&D Project (2016YFD0500202), the National Natural Science Foundation of China $(31872473,31602057,31802212$, 31872477), the Independent Innovation of Agricultural Sciences in Jiangsu Province [Grant Number CX (18)3018] and a project funded by the Priority Academic Program Development of Jiangsu Higher Education (PAPD). Jiangsu Provincial Natural Science Foundation of China (BK20160456). The funding 
bodies had no role in study design, data collection and analysis, decision to publish or preparation of the manuscript.

Author contributions.

YD, FY, QW, NX, and YX performed the experiments. YD, SC, TQ, and DP conceived and designed the experiments. YD and DP prepared the manuscript.

\section{Acknowledgements}

Not applicable

\section{References}

1. Long JS, Mistry B, Haslam SM, Barclay WS: Host and viral determinants of influenza A virus species specificity. Nat Rev Microbiol 2019, 17:67-81.

2. Peacock T, James J, Sealy JE, Iqbal M: A Global Perspective on H9N2 Avian Influenza Virus. Viruses 2019, 11.

3. Lai S, Qin Y, Cowling BJ, Ren X, Wardrop NA, Gilbert M, Tsang TK, Wu P, Feng L, Jiang H, et al: Global epidemiology of avian influenza A H5N1 virus infection in humans, 1997-2015: a systematic review of individual case data. Lancet Infect Dis 2016, 16:e108-e118.

4. Yamayoshi S, Kiso M, Yasuhara A, Ito M, Shu Y, Kawaoka Y: Enhanced Replication of Highly Pathogenic Influenza A(H7N9) Virus in Humans. Emerg Infect Dis 2018, 24:746-750.

5. Iwasaki A, Pillai PS: Innate immunity to influenza virus infection. Nat Rev Immunol 2014, 14:315-328.

6. Schneider WM, Chevillotte MD, Rice CM: Interferon-stimulated genes: a complex web of host defenses. Annu Rev Immunol 2014, 32:513-545.

7. Lazear HM, Schoggins JW, Diamond MS: Shared and Distinct Functions of Type I and Type III Interferons. Immunity 2019, 50:907-923.

8. Schoggins JW, Rice CM: Interferon-stimulated genes and their antiviral effector functions. Curr Opin Virol 2011, 1:519-525.

9. Koerner I, Kochs G, Kalinke U, Weiss S, Staeheli P: Protective role of beta interferon in host defense against influenza A virus. J Virol 2007, 81:2025-2030.

10. Price GE, Gaszewska-Mastarlarz A, Moskophidis D: The role of alpha/beta and gamma interferons in development of immunity to influenza A virus in mice. J Virol 2000, 74:3996-4003.

11. Xia C, Vijayan M, Pritzl CJ, Fuchs SY, McDermott AB, Hahm B: Hemagglutinin of Influenza A Virus Antagonizes Type I Interferon (IFN) Responses by Inducing Degradation of Type I IFN Receptor 1. J Virol 2015, 90:2403-2417.

12. Wei H, Wang S, Chen Q, Chen Y, Chi X, Zhang L, Huang S, Gao GF, Chen JL: Suppression of interferon lambda signaling by SOCS-1 results in their excessive production during influenza virus infection. Plos Pathog 2014, 10:e1003845. 
13. Tang $Y$, Wu P, Peng D, Wang X, Wan H, Zhang P, Long J, Zhang W, Li Y, Wang W, et al: Characterization of duck H5N1 influenza viruses with differing pathogenicity in mallard (Anas platyrhynchos) ducks. Avian Pathol 2009, 38:457-467.

14. Sun Z, Qin T, Meng F, Chen S, Peng D, Liu X: Development of a multiplex probe combination-based one-step real-time reverse transcription-PCR for NA subtype typing of avian influenza virus. Sci Rep 2017, 7:13455.

15. Zhu Y, Yang Y, Liu W, Liu X, Yang D, Sun Z, Ju Y, Chen S, Peng D, Liu X: Comparison of biological characteristics of H9N2 avian influenza viruses isolated from different hosts. Arch Virol 2015, 160:917-927.

16. Lee Y, Hyung SW, Jung HJ, Kim HJ, Staerk J, Constantinescu SN, Chang EJ, Lee ZH, Lee SW, Kim HH: The ubiquitin-mediated degradation of Jak1 modulates osteoclastogenesis by limiting interferonbeta-induced inhibitory signaling. Blood 2008, 111:885-893.

17. Garcia-Sastre A: Induction and evasion of type I interferon responses by influenza viruses. Virus Res 2011, 162:12-18.

18. Porritt RA, Hertzog PJ: Dynamic control of type I IFN signalling by an integrated network of negative regulators. Trends Immunol 2015, 36:150-160.

19. Croker BA, Kiu H, Nicholson SE: SOCS regulation of the JAK/STAT signalling pathway. Semin Cell Dev Biol 2008, 19:414-422.

20. Pothlichet $\mathrm{J}$, Chignard $\mathrm{M}$, Si-Tahar M: Cutting edge: innate immune response triggered by influenza $\mathrm{A}$ virus is negatively regulated by SOCS1 and SOCS3 through a RIG-I/IFNAR1-dependent pathway. $J$ Immunol 2008, 180:2034-2038.

21. Boyle K, Zhang JG, Nicholson SE, Trounson E, Babon JJ, McManus EJ, Nicola NA, Robb L: Deletion of the SOCS box of suppressor of cytokine signaling 3 (SOCS3) in embryonic stem cells reveals SOCS box-dependent regulation of JAK but not STAT phosphorylation. Cell Signal 2009, 21:394-404.

22. Zhang JG, Farley A, Nicholson SE, Willson TA, Zugaro LM, Simpson RJ, Moritz RL, Cary D, Richardson $R$, Hausmann $G$, et al: The conserved SOCS box motif in suppressors of cytokine signaling binds to elongins $\mathrm{B}$ and $\mathrm{C}$ and may couple bound proteins to proteasomal degradation. Proc Natl Acad Sci U $S$ A 1999, 96:2071-2076.

23. Mesev EV, LeDesma RA, Ploss A: Decoding type I and III interferon signalling during viral infection. Nat Microbiol 2019, 4:914-924.

24. Ghoreschi K, Laurence A, O'Shea JJ: Janus kinases in immune cell signaling. Immunol Rev 2009, 228:273-287.

25. Xia C, Anderson P, Hahm B: Viral dedication to vigorous destruction of interferon receptors. Virology 2018, 522:19-26.

26. Fuchs SY: Ubiquitination-mediated regulation of interferon responses. Growth Factors 2012, 30:141148.

27. Weihua X, Ramanujam S, Lindner DJ, Kudaravalli RD, Freund R, Kalvakolanu DV: The polyoma virus T antigen interferes with interferon-inducible gene expression. Proc Natl Acad Sci U S A 1998, 
95:1085-1090.

28. Li D, Wei J, Yang F, Liu HN, Zhu ZX, Cao WJ, Li S, Liu XT, Zheng HX, Shu HB: Foot-and-mouth disease virus structural protein VP3 degrades Janus kinase 1 to inhibit IFN-gamma signal transduction pathways. Cell Cycle 2016, 15:850-860.

29. Wu Y, Liu Q, Zhou J, Xie W, Chen C, Wang Z, Yang H, Cui J: Zika virus evades interferon-mediated antiviral response through the co-operation of multiple nonstructural proteins in vitro. Cell Discov 2017, 3:17006.

30. Miller DM, Zhang Y, Rahill BM, Waldman WJ, Sedmak DD: Human cytomegalovirus inhibits IFNalpha-stimulated antiviral and immunoregulatory responses by blocking multiple levels of IFN-alpha signal transduction. J Immunol 1999, 162:6107-6113.

31. Uetani K, Hiroi M, Meguro T, Ogawa H, Kamisako T, Ohmori Y, Erzurum SC: Influenza A virus abrogates IFN-gamma response in respiratory epithelial cells by disruption of the Jak/Stat pathway. Eur J Immunol 2008, 38:1559-1573.

32. Fenner JE, Starr R, Cornish AL, Zhang JG, Metcalf D, Schreiber RD, Sheehan K, Hilton DJ, Alexander WS, Hertzog PJ: Suppressor of cytokine signaling 1 regulates the immune response to infection by a unique inhibition of type I interferon activity. Nat Immunol 2006, 7:33-39.

33. Qing Y, Costa-Pereira AP, Watling D, Stark GR: Role of tyrosine 441 of interferon-gamma receptor subunit 1 in SOCS-1-mediated attenuation of STAT1 activation. J Biol Chem 2005, 280:1849-1853.

34. Liau N, Laktyushin A, Lucet IS, Murphy JM, Yao S, Whitlock E, Callaghan K, Nicola NA, Kershaw NJ, Babon JJ: The molecular basis of JAK/STAT inhibition by SOCS1. Nat Commun 2018, 9:1558.

35. Limnander A, Danial NN, Rothman PB: v-Abl signaling disrupts SOCS-1 function in transformed pre-B cells. Mol Cell 2004, 15:329-341.

36. Qiu X, Guo G, Chen K, Kashiwada M, Druker BJ, Rothman PB, Chen JL: A requirement for SOCS-1 and SOCS-3 phosphorylation in Bcr-Abl-induced tumorigenesis. Neoplasia 2012, 14:547-558.

37. Akhtar LN, Benveniste EN: Viral exploitation of host SOCS protein functions. J Viro/ 2011, 85:19121921.

38. O'Leary CE, Riling CR, Spruce LA, Ding H, Kumar S, Deng G, Liu Y, Seeholzer SH, Oliver PM: Ndfipmediated degradation of Jak1 tunes cytokine signalling to limit expansion of CD4+ effector T cells. Nat Commun 2016, 7:11226.

\section{Figures}


a
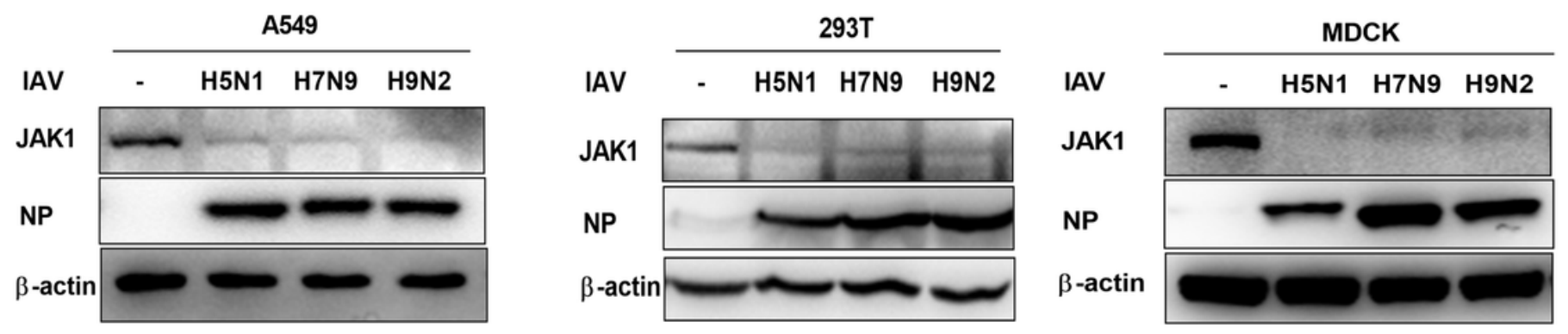

b

\section{C}

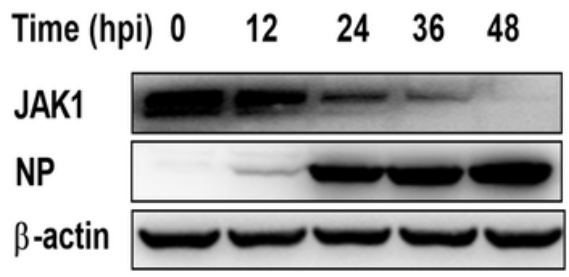

293T

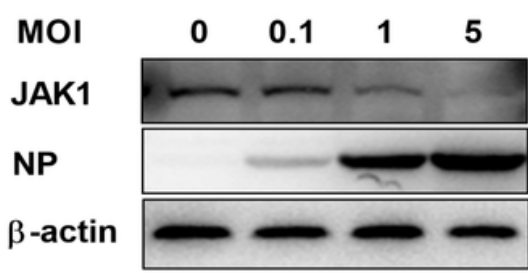

293T

\section{Figure 1}

IAV induces the degradation of JAK1. (a) A549 cells, 293T cells and MDCK cells were infected or uninfected (Mock) with indicated IAV (A/mallard/Huadong/S/2005 (H5N1), A/chicken/Jiangsu/WJ14/2015 (H7N9) and A/chicken/Taixing/10/2010 (H9N2)) at an MOI of 1 for 24 hours, The protein levels of JAK1 and viral NP were analyzed by Western blotting. The protein level of $\beta$-actin was used as internal loading control. (b) 293T cells were left uninfected or infected with IAV influenza A/mallard/Huadong/S/2005 (H5N1), which was used for the majority of the following experiments unless indicated otherwise, at an $\mathrm{MOI}$ of 1 for the indicated times. The levels of JAK1, viral NP and $\beta$-actin were analyzed by Western blotting. (c) 293T cells were left uninfected or infected with IAV at the indicated $\mathrm{MOI}$ for $24 \mathrm{~h}$. The protein levels of JAK1, viral NP and $\beta$-actin were analyzed using Western blotting. 
a

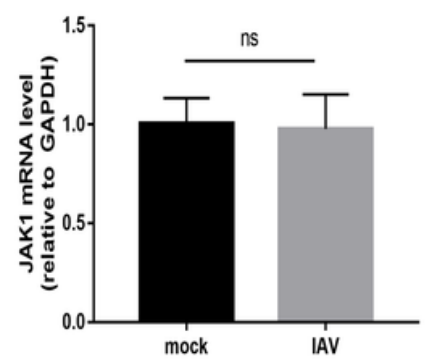

A549

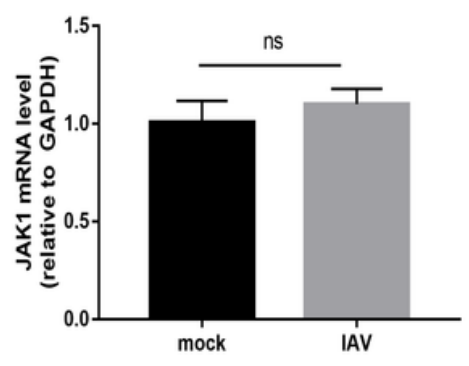

293T

b

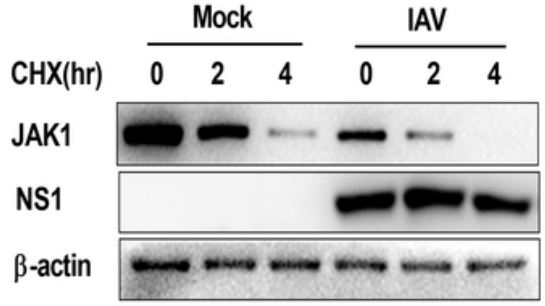

293T
C

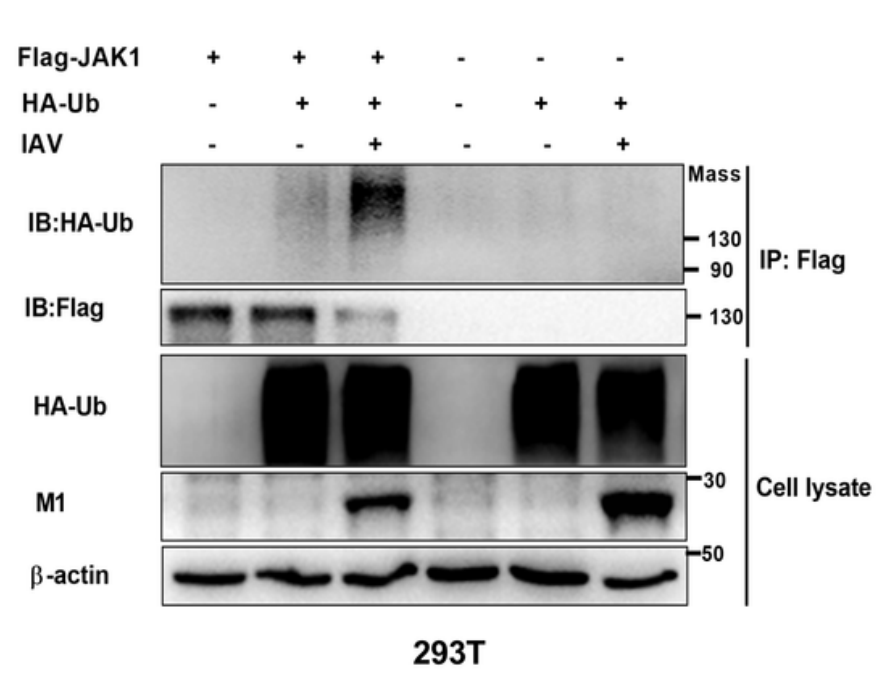

d

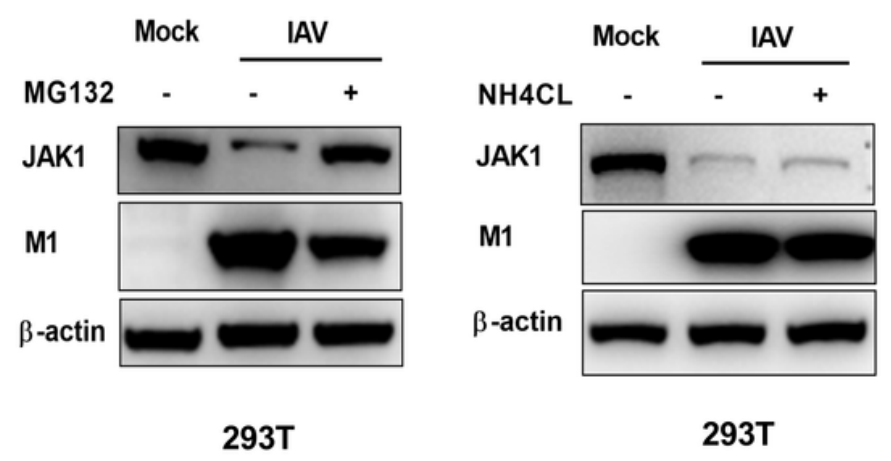

\section{Figure 2}

IAV infection induces ubiquitination and proteasome-mediated degradation of JAK1. (a) 293T cells and A549 cells were left uninfected or infected with IAV at an MOI of 1 . The relative mRNA levels of JAK1 were analyzed by real-time qPCR at $24 \mathrm{hpi}$. Date are means plus standard deviation for triplicate samples. Ns, not significant. (b) 293T cells were uninfected or infected with IAV at an MOI of 1 . At $12 \mathrm{hpi}$, the cells were treated with solvent or cycloheximide $(\mathrm{CHX}, 50 \mu \mathrm{g} / \mathrm{ml})$ for the indicated times. The levels of JAK1, viral NS1 and $\beta$-actin were analyzed using Western blotting. (c) 293T cells were transfected with Flag-tagged JAK1 and HA-tagged ubiquitin, $24 \mathrm{~h}$ post transfection, cells were left uninfected or infected with IAV at an $\mathrm{MOI}$ of 1 as indicated for additional 18 hours. Cell lysis was subjected to IP and Western blotting. The ubiquitination of immunoprecipitated Flag-JAK1 was analyzed by Western blotting using anti-HA tag antibody. The protein levels of Flag-JAK1, HA-Ub, $\beta$-actin, and viral M1 in the whole-cell lysates were also analyzed using Western blotting. (d) 293T cells were infected with IAV at an MOI of 1. At 18 hours post infection, cells were treated with indicated inhibitors or solvent (dimethyl sulfoxide [DMSO]) for an additional $6 \mathrm{~h}$. The protein levels of JAK1, viral M1, and $\beta$-actin were analyzed using Western blotting. 
a

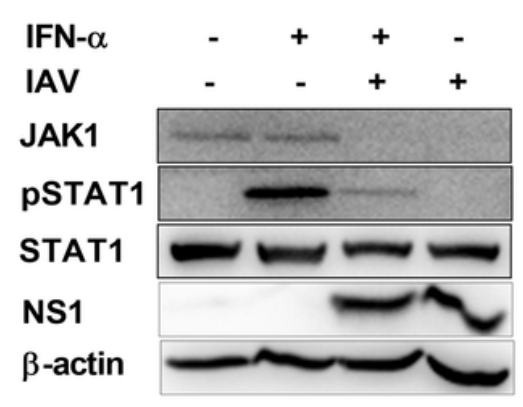

b

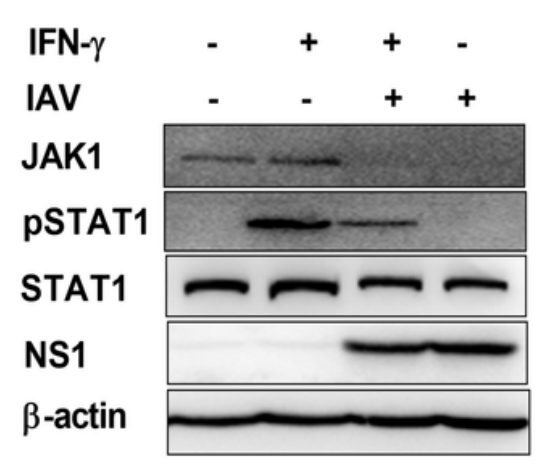

C

ISG56 mRNA
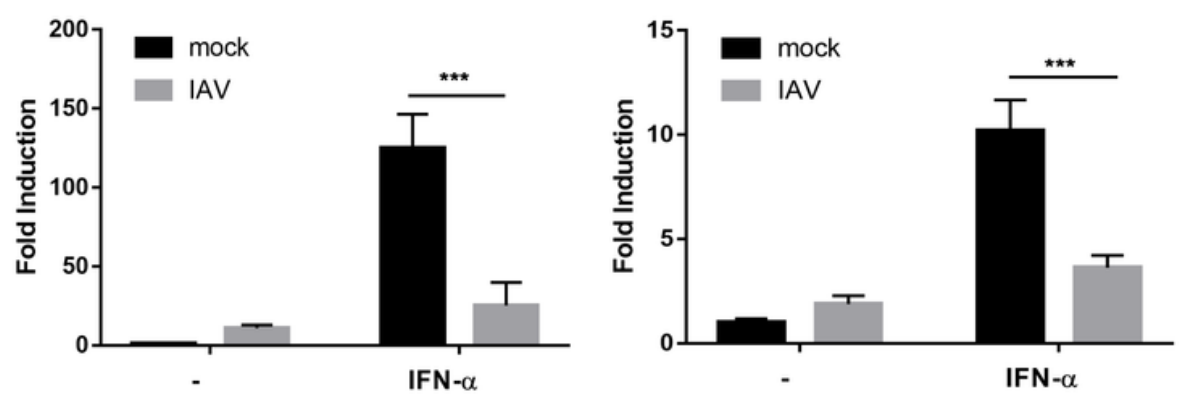

d

TAP-1 mRNA

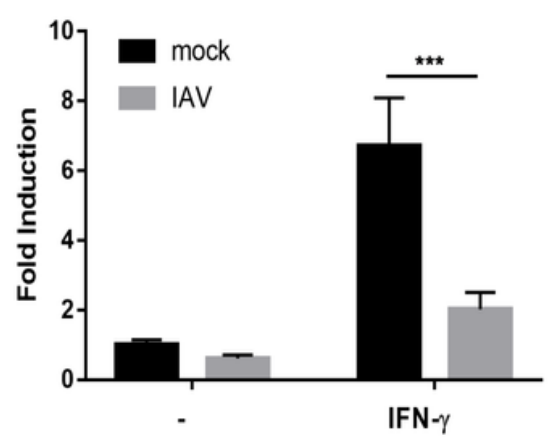

LMP-2 mRNA

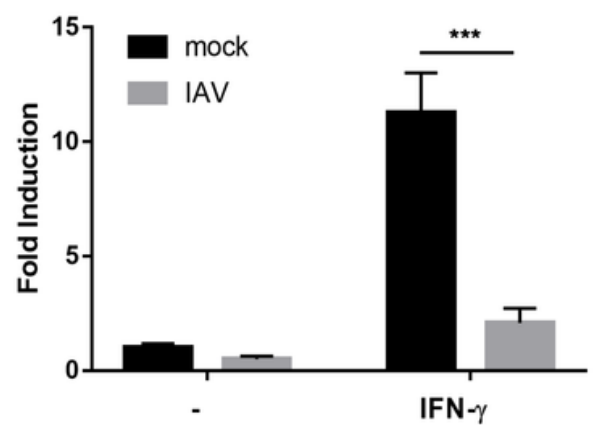

Figure 3

IAV infection reduces responsiveness to IFNs. (a) 293T cells were left uninfected (Mock) or infected with IAV at an MOI of 1.24 hours post infection, cells were left untreated (-) or treated with human IFN-a (1000 $\mathrm{U} / \mathrm{ml}$ ) for $1 \mathrm{~h}$. The levels of JAK1, pSTAT1, STAT1, viral NS1, and $\beta$-actin were detected using Western blotting. (b) 293T cells were left uninfected (Mock) or infected with IAV at an MOI of 1. At $24 \mathrm{hpi}$, cells were left untreated (-) or treated with human IFN- $\gamma(1000 \mathrm{U} / \mathrm{ml})$ for $1 \mathrm{~h}$. The levels of JAK1, pSTAT1, STAT1, viral NS1, and $\beta$-actin were detected using Western blotting. (c) 293T cells were left uninfected (Mock) or infected with IAV at an $\mathrm{MOI}$ of 1 . At $24 \mathrm{hpi}$, cells were left untreated (-) or treated with human IFN-a $(1000 \mathrm{U} / \mathrm{ml})$ for $24 \mathrm{~h}$. The relative mRNA levels of Mx1 and ISG56 were analyzed using real-time qPCR. The error bars represent the means plus standard deviations for three independent experiments. $\star \star \star \mathrm{P}<.0001$. (d) 293T cells were left uninfected (Mock) or infected with IAV at an MOI of 1. At $24 \mathrm{hpi}$, cells were left untreated (-) or treated with IFN- $(1000 \mathrm{U} / \mathrm{ml})$ for $6 \mathrm{~h}$. The relative mRNA levels of TAP-1 and LMP-2 were analyzed using real-time qPCR. The error bars represent the means plus standard deviations for three independent experiments. ${ }^{\star} * \star \mathrm{P}<.0001$. 
a

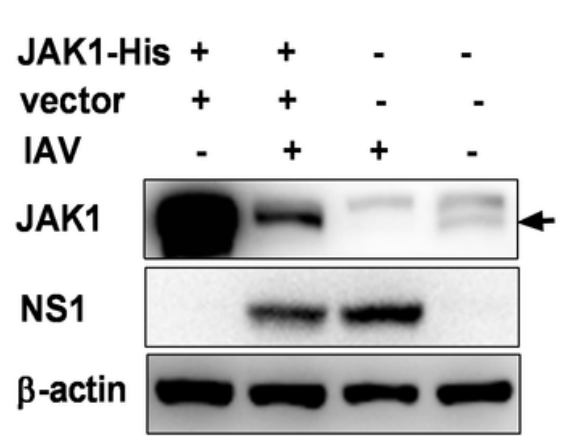

d

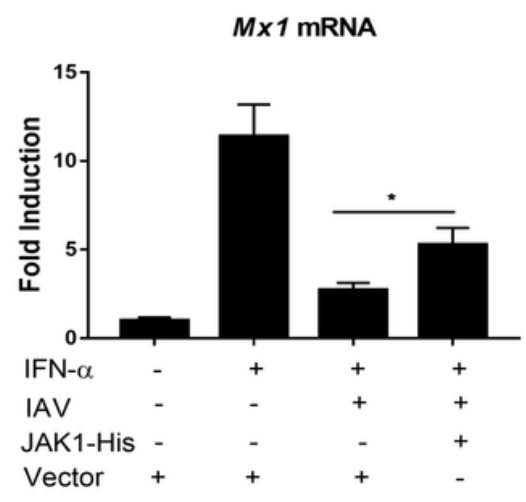

b

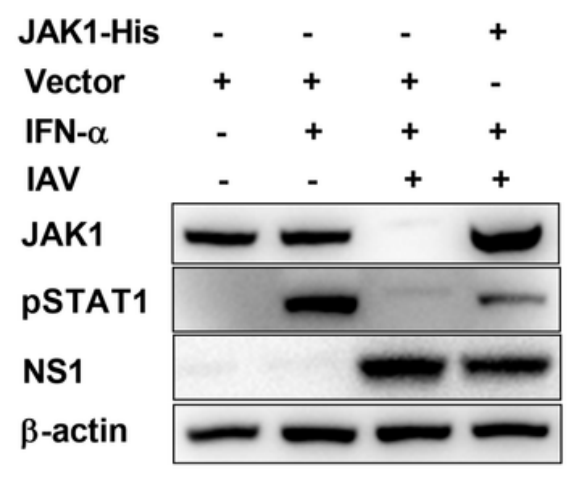

C

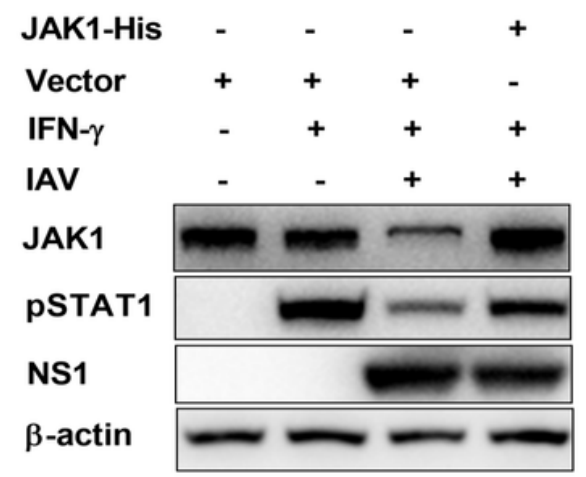

e

TAP-1 mRNA

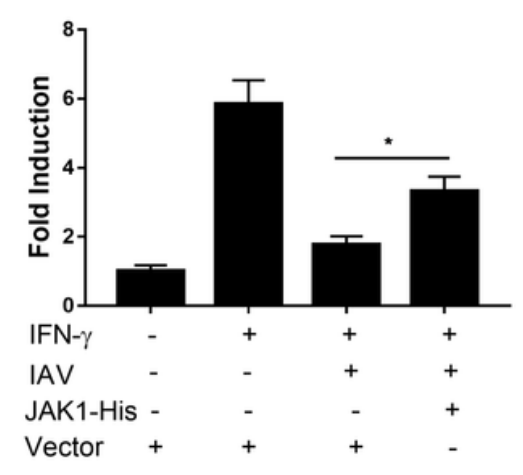

\section{Figure 4}

Rescuing JAK1 overexpression enhances cellular responses to type I and type II IFNs. (a) 293T cells were transfected with control vector or plasmid encoding JAK1-His. At $24 \mathrm{~h}$ post-transfection, cells were infected with IAV at an MOI of 1 for an additional $24 \mathrm{~h}$. The level of JAK1, viral NS1, and $\beta$-actin were detected using Western blotting. (b) 293T cells were transfected with control vector or plasmid encoding JAK1-His. At $24 \mathrm{~h}$ post transfection, cells were infected with IAV at an MOI of 1 . At $24 \mathrm{hpi}$, cells were left untreated (-) or treated with human IFN-a $(1000 \mathrm{U} / \mathrm{ml})$ for 1 hour. The level of JAK1, pSTAT1, viral NS1, and $\beta$-actin were detected using Western blotting. (c) 293T cells were transfected with control vector or plasmid encoding JAK1-His. At $24 \mathrm{~h}$ post-transfection, cells were infected with IAV at an MOI of 1. At 24 hpi, cells were left untreated (-) or treated with human IFN-y $(1000 \mathrm{U} / \mathrm{ml})$ for $1 \mathrm{~h}$ The level of JAK1, PSTAT1, viral NS1, and $\beta$-actin were detected using Western blotting. (d) 293T cells were transfected with control vector or plasmid encoding JAK1-His. 293T cells were left uninfected (Mock) or infected with IAV at an $\mathrm{MOI}$ of 1 . At $24 \mathrm{hpi}$, cells were left untreated (-) or treat with human IFN-a $(1000 \mathrm{U} / \mathrm{ml})$ for 24 hours for $6 \mathrm{~h}$. The relative mRNA levels of Mx 1 were analyzed using real-time qPCR. The error bars represent the means plus standard deviations for three independent experiments. *, $p \leq 0.05$. (e) 293T cells were transfected with control vector or plasmid encoding JAK1-His. 293T cells were left uninfected (Mock) or infected with IAV at an $\mathrm{MOI}$ of 1 . At $24 \mathrm{hpi}$, cells were left untreated (-) or treat with human IFN-a (1000 $\mathrm{U} / \mathrm{ml}$ ) for 6 hours. The relative mRNA levels of TAP- 1 were analyzed using real-time qPCR. The error bars represent the means plus standard deviations for three independent experiments. ${ }^{*}, p \leq 0.05$. 


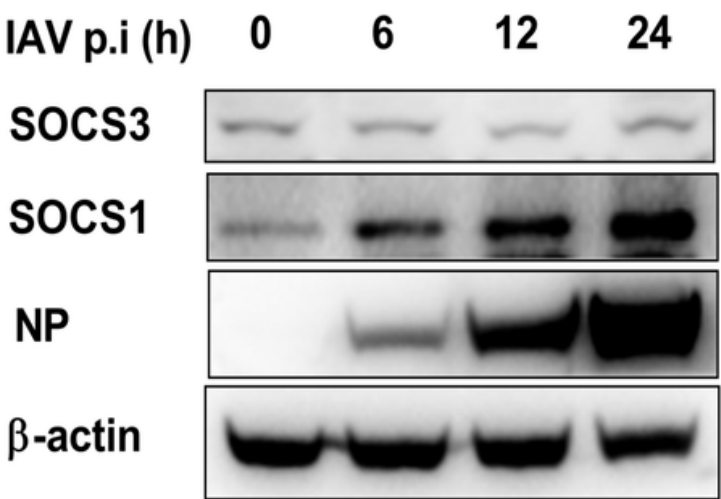

C

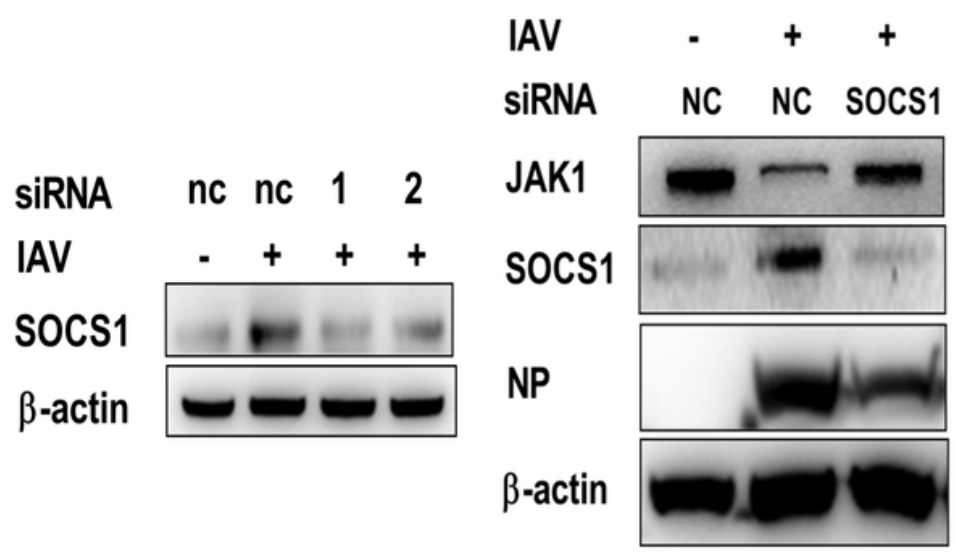

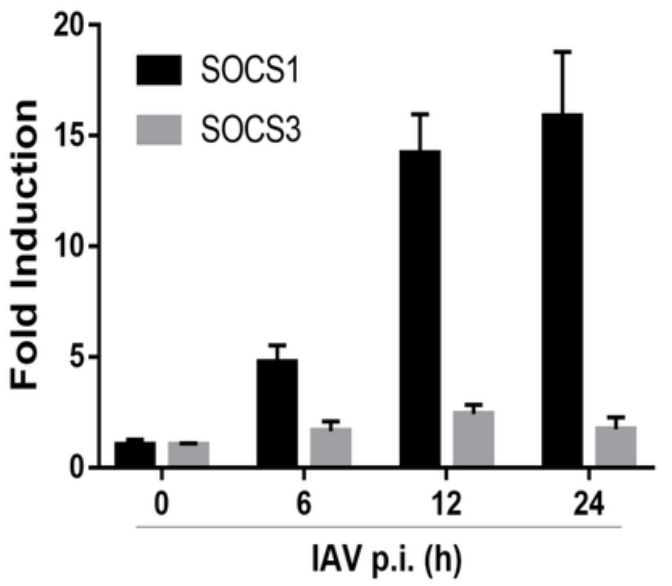

d

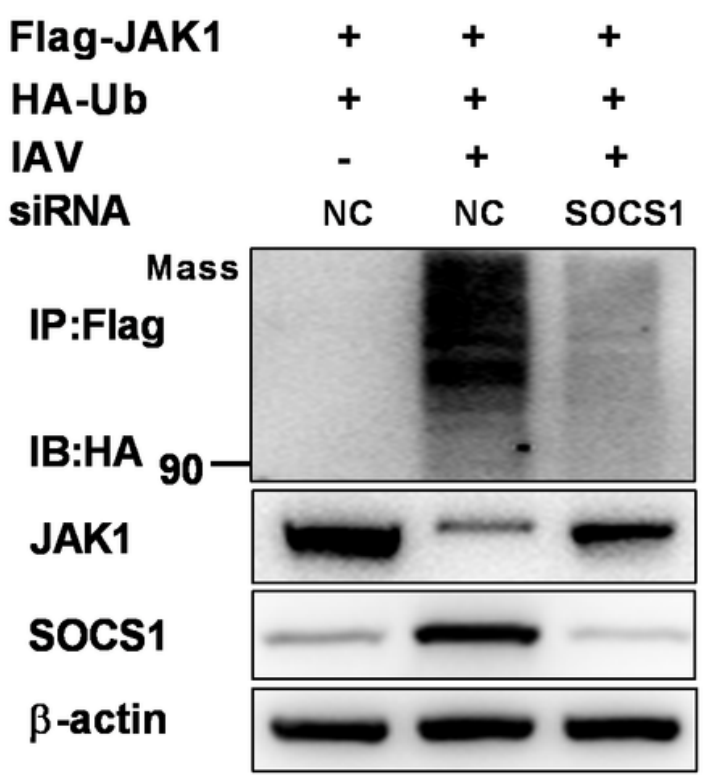

Figure 5

IAV infection induces the expression of SOCS1, resulting in JAK1 degradation. (a) 293T cells were left uninfected or infected with IAV at an MOI of 1 for the indicated times. The protein levels of SOCS1, SOCS3, viral NP and $\beta$-actin were analyzed using Western blotting. (b) 293T cells were left uninfected or infected with IAV at an MOI of 1 for the indicated times. The mRNA levels of SOCS1, SOCS3 were analyzed using real-time qPCR. The error bars represent the means plus standard deviations for three independent experiments. (c) 293T cells were transfected with siRNAs 1 and 2 targeting SOCS1 or NC (negative control) siRNA. Twenty-four hours later, cells were left uninfected or infected with IAV at an MOI of 1 . The protein levels of SOCS1 and $\beta$-actin were analyzed by Western blotting at $24 \mathrm{hpi}$, and the protein level of SOCS1, JAK1, viral NP and $\beta$-actin were detected. (d) 293T cells were transfected with siRNA targeting SOCS1 or NC siRNA as indicated. Six hours later, cells were transfected with Flag-tagged JAK1 
and HA-tagged ubiquitin. Twenty-four hours post-transfection, cells were left uninfected or infected with IAV at an MOI of 1 . At $18 \mathrm{~h}$ post infection, the cells were harvested and subjected to immunoprecipitation. The ubiquitination of immunoprecipitated Flag-JAK1 was analyzed using Western blotting using anti-HA tag antibody. The protein level of Flag-JAK1, SOCS1 and $\beta$-actin in the whole-cell lysates were also analyzed using Western blotting.

a

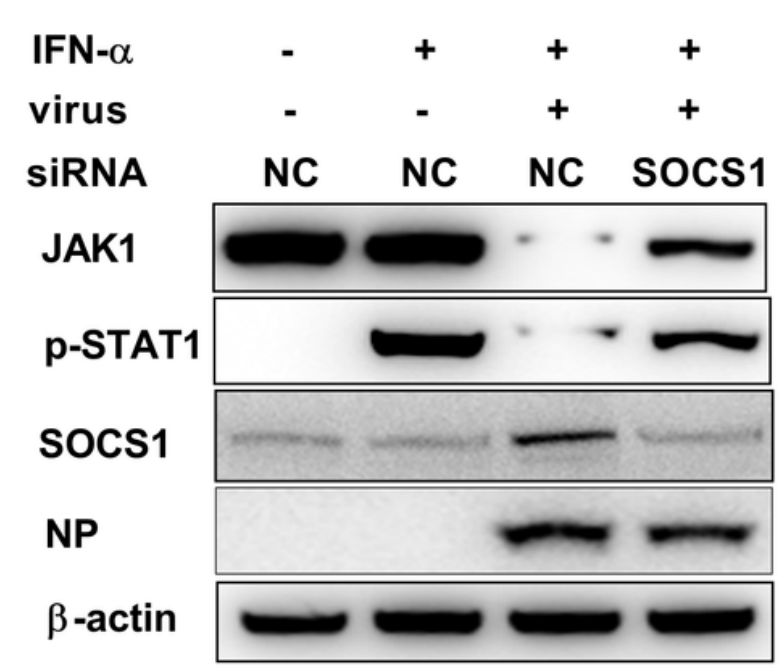

C

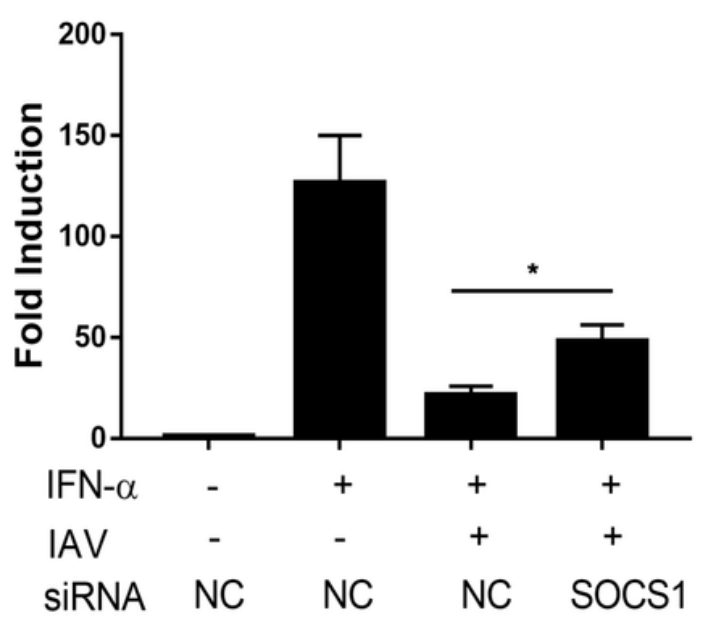

b

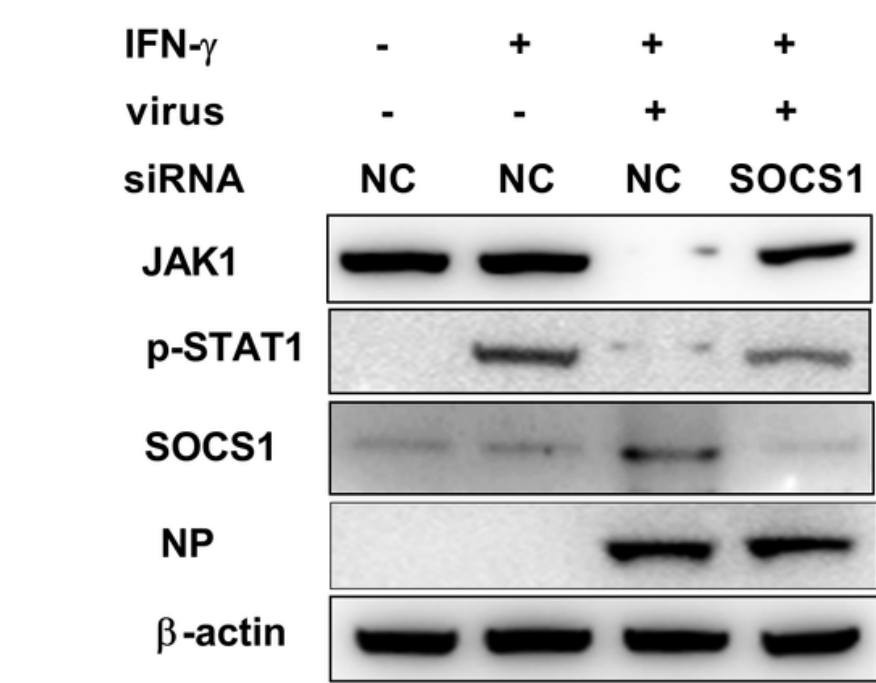

d

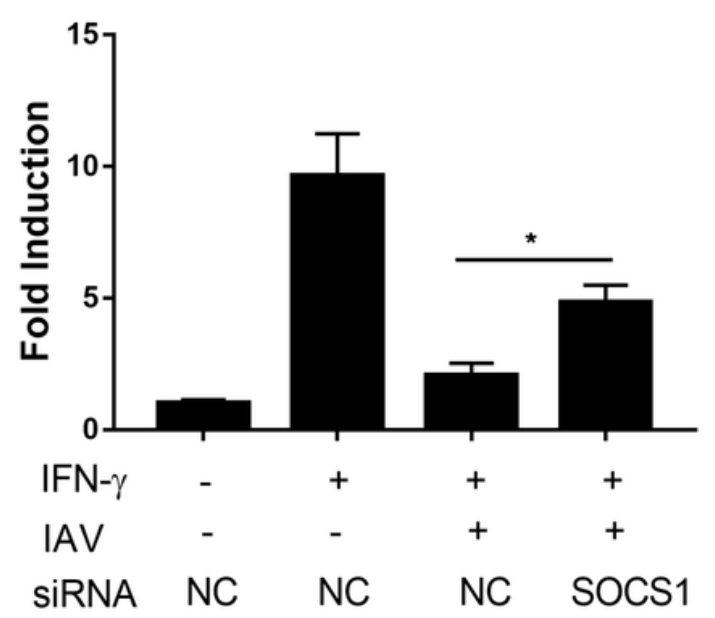

Figure 6

SOCS1 mediates the inhibition of IFNs response. ( $a$ and $b$ ) 293T cells were transfected with siRNA targeting SOCS1 or NC siRNA. 24 hours later, cells were left uninfected or infected with IAV at an MOI of 1. 2 Twenty-four hours post infection, cells were left untreated (-) or treated with human IFN- $\mathrm{a}(1000 \mathrm{U} / \mathrm{ml})$ (a) or IFN-y $(1000 \mathrm{U} / \mathrm{ml})$ (b) for 1 hour, and the protein level of JAK1, pSTAT1, SOCS1, viral NP and $\beta$-actin were analyzed using Western blotting. (c and d) 293T cells were transfected with siRNA targeting 
SOCS1or NC siRNA. Six hours later, 293T cells were left uninfected or infected with IAV at an MOI of 1. At $24 \mathrm{hpi}$, cells were left untreated (-) or treated with human IFN-a $(1000 \mathrm{U} / \mathrm{ml})$ for 24 hours or IFN- $\mathrm{Y}(1000$ $\mathrm{U} / \mathrm{ml}$ ) for $6 \mathrm{~h}$. The relative mRNA levels of ISG56 (c) or LMP-2 (d) were analyzed using real-time qPCR. The error bars represent the means plus standard deviations for three independent experiments. ${ }^{*}, \mathrm{p} \leq$ 0.05 . 